\title{
La orientación para la salud mental: Reflexiones para delimitar un campo de intervención profesional
}

\author{
Mental Health Counseling: Thoughts on Defining a Professional Intervention field
}

\author{
Ana Victoria Garita Pulido' \\ Colegio Técnico Profesional de Pavas \\ San José, Costa Rica \\ avictoria@gmail.com
}

Recibido 31 de enero de 2013 • Corregido 10 de marzo de 2013 • Aceptado 13 de marzo de 2013

Resumen. El propósito de este artículo es presentar algunas reflexiones sobre una serie de argumentos que permiten delimitar la salud mental como campo de intervención para la orientación. Se hace alusión al posicionamiento epistemológico que se asume para comprender la salud, centrado en una perspectiva contextual, y se propone el modelo de promoción de la salud para sustentar la acción orientadora. Se presentan las razones que circunscriben la intervención a la promoción de la salud mental vinculadas a los referentes teóricos y metodológicos propios de la disciplina. A la vez, se sugieren aspectos a considerar en el abordaje individual y grupal, así como para ampliar la práctica profesional con un ejercicio eficiente de la persona orientadora en su rol como promotora de salud mental.

Palabras claves. Orientación, salud, teoría ecológica, intervención, promoción de la salud mental, fortalezas, Costa Rica, Revista Electrónica Educare.

Abstract. The purpose of this paper is to provide some thoughts on a series of points of view that will help us in defining mental health as an intervention field of counseling. Reference is made to the epistemological positioning adopted to understand health, which is focused on a contextual perspective. A health promotion model is proposed to support the counseling activity. The reasons for limiting the intervention to mental health promotion, which are related to theoretical and methodological references inherent to the discipline, are also explained. Recommendations are made on the aspects to be considered for both individual and collective approaches and for extending the professional practice of counselors with an efficient performance as mental health promoters.

Keywords. Counseling, health, ecological theory, intervention, mental health promotion, strengths, Costa Rica, Educare e-Journal.

1 Magistra en Orientación de la Universidad de Costa Rica. Bachiller y licenciada en Ciencias de la Educación con énfasis en Orientación de la Universidad Católica de Costa Rica. Ha laborado como orientadora en centros de educación secundaria pública y privada y como docente de la carrera de Orientación Educativa de la Universidad Católica de Costa Rica. Actualmente labora para el Ministerio de Educación Pública en el Colegio Técnico Profesional de Pavas. 
URL: http://www.una.ac.cr/educare

CORREO: educare@una.cr

\section{La salud como campo de acción de la orientación}

La salud como campo de actuación para la orientación supone acciones específicas que favorezcan el bienestar físico, psicológico y social de las poblaciones que se atienden. El propósito de este artículo es presentar algunos argumentos que permitan delimitar este campo de actuación y la intervención orientadora, vinculados a un posicionamiento contextual de la salud, el modelo de promoción de salud y el rol de la persona orientadora como promotora de salud mental.

Las realidades sociales actuales plantean, a los diversos actores sociales, nuevas demandas de sentido relacionadas con la comprensión de los contextos sociales complejos en que las personas se desarrollan. En el caso de la orientación, dichas demandas conllevan a focalizar en acciones que promuevan el bienestar personal y colectivo, independientemente de las circunstancias que caracterizan dichos escenarios sociales.

Las realidades y condiciones sociales actuales que vivencian los distintos grupos de poblaciones vinculadas a factores de tipo político, económico, laboral, familiar, entre otros, representan elementos favorecedores u obstaculizadores de su desarrollo, según el lugar y posición que ocupen en la estructura social, por cuanto estos factores "(...) permean todas las áreas de la vida humana, y vuelven la existencia más susceptible de enfrentar problemas, lo que a su vez deviene en mayor riesgo para la salud individual y colectiva" (Bonilla, 1993, p. 55).

El estilo de vida de las personas tiene implícito un riesgo para su salud cuando este se caracteriza por realidades y condiciones de desigualdad y exclusión, por razones de género, cultura, discapacidad, religión, etnia, preferencia sexual, enfermedad o pobreza.

Estas condiciones deben ser tomadas en cuenta en cualquier política de orientación, pues las problemáticas psicosociales pueden afectar la salud mental de los grupos poblacionales, lo cual debe dar origen a un redireccionamiento de las estrategias y de los diversos centros y servicios de Orientación en el mundo (Gavilán, 2006).

Este hecho implica dar respuestas oportunas, para que las personas logren enfrentar adecuadamente sus circunstancias vitales y favorecer el poder de acción sobre su bienestar personal. Con lo cual puede decirse que la salud cobra relevancia como campo de intervención para la disciplina de orientación, pues cuenta con referentes teóricos, metodológicos e instrumentales que le posibilitan rescatar las fortalezas y habilidades que posee la persona, a pesar de su problema, así como para desarrollar nuevas habilidades para enfrentar sus vidas (Bonilla, 1993).

La persona orientadora deberá dirigir sus acciones al mejoramiento de la calidad de vida de las poblaciones que atiende en términos de su bienestar físico, emocional y social (Gavilán, 2006), más que a una acción aislada y asistencialista centrada en el problema.

La salud, al ser incluida dentro de los programas y proyectos que se ejecuten independientemente del contexto y población que se aborde, supone para la persona orientadora un posicionamiento integral, claro y coherente en el cual sustentar su accionar en este campo de intervención. 


\section{El posicionamiento epistemológico para la comprensión de la salud}

Al incursionar en la salud como campo de intervención, se requiere conceptuarla, de manera que se tenga conciencia del posicionamiento epistemológico que subyace en su accionar. Como se indicó, los procesos sociales y las circunstancias vitales vinculadas a aspectos culturales, políticos, económicos, entre otros, son elementos que intervienen en el desarrollo humano y, por ende, en el bienestar personal, familiar y colectivo. Esto demanda asumir una posición paradigmática con respecto a la salud, centrada en una relación dialéctica entre la persona y los elementos constitutivos de su entorno.

Para Marks, Murray y Evans (2008), el modelo biopsicosocial representa una posición paradigmática que comprende la salud como un proceso complejo en el que intervienen múltiples factores más que la simple idea de la ausencia de enfermedad; de ahí que definen la salud como "(...) un estado de bienestar con atributos físicos, culturales, psicosociales, económicos y espirituales (...)" (Marks et al., 2008, p. 4).

Esta definición implica que hay situaciones externas que inciden en la salud, por lo que no se puede garantizar un bienestar total, pues la ausencia de enfermedad no significa que no se experimenten otras situaciones que limiten el bienestar personal.

El modelo biopsicosocial propone que para responder adecuadamente a las necesidades relacionadas con una enfermedad, es necesario tener en consideración tanto los factores biológicos como psicológicos, sociales y espirituales que concurren en las personas y que inciden en su estado de salud (Morago, 2006).

Aspectos referidos a las oportunidades y amenazas del medio, el lugar que se ocupe en la estructura social y las representaciones sociales inherentes, los recursos materiales y económicos disponibles, las condiciones de vivienda, las redes sociales con que se cuente, los pensamientos y las emociones que provocan dichas situaciones, entre otros, tienen implicaciones importantes en la salud.

Por tanto, la salud ha de ser entendida desde una perspectiva sistémica, ya que los diversos componentes que intervienen en esta -biológico, axiológico, cognitivo, afectivo, interpersonal, social, ambiental- están interrelacionados, hay causalidad circular, y los cambios en cualquiera de estos elementos afecta la totalidad, es decir, al individuo (Oblitas, 2006).

Desde este modelo no puede comprenderse la salud como un estado que se alcanza mediante el abordaje de cada uno de los elementos que intervienen de manera aislada ni tampoco como la simple suma de cada uno. Más bien, brinda explicaciones integradoras, tanto de la salud como de la enfermedad, basadas en la valoración de aspectos psicológicos, biológicos y sociales que se encuentran interrelacionados (González y García, 1994). 
URL: http://www.una.ac.cr/educare

CORREO: educare@una.cr

Esta conceptuación implica trascender la idea simplista y su connotación de ausencia de enfermedad, a la idea de salud como proceso, como bienestar, como funcionamiento positivo, como calidad de vida en general (Flórez, 2007), que se sustenta en la interrelación existente entre los elementos antes mencionados.

En esta línea, Flórez (2007) y Marks et al. (2008) puntualizan los elementos que interactúan en el proceso de salud:

- Las características biológicas: predisposición genética, enfermedades adquiridas, cambios bioquímicos, entre otros.

- Losfactores conductuales:estos caracterizan las pautasindividuales decomportamiento asociados al estilo de vida de las personas como su forma general de vivir, las condiciones de vida, las metas que fija para su vida, los factores de riesgo ante la enfermedad o protección de la salud, y las decisiones que toman en su contexto sociocultural, biológico y social.

- Condiciones sociales: influencias culturales, relaciones familiares y apoyo social.

- Factores psicológicos: autoestima, manejo del estrés, resolución de conflictos, creencias acerca de la salud, entre otros.

La interrelación y la sinergia entre estos factores conllevan la percepción de bienestar y de vivir una vida con calidad. Comprender las condiciones personales y recursos sociales, ambientales y contextuales les permite a las personas alcanzar su bienestar integral, ya que pueden visualizar los elementos que favorecen o limitan su desarrollo. Esto hace necesario posicionarse en un marco de referencia integrador que ratifique su comprensión desde una perspectiva contextual.

De manera que al visualizar todos los componentes del ser humano, se comprende a la persona como un todo en interacción con el contexto. La teoría ecológica propuesta por Bronfenbrenner hace referencia a un marco ecológico para la comprensión de "(...) la interacción e interdependencia de los humanos (como individuos, grupos o sociedades) y su ambiente que son parte inseparable de un todo mayor (...)" (López y Escudero, 2003, p. 73).

Esto supone una relación dialéctica, en que confluye la doble naturaleza de la persona: como ser individual y social, en continua interacción con otras personas y ambientes, que inciden en su desarrollo.

Según Papalia, Wendkos y Duskin (2001, p. 42) “(...) de acuerdo con Bronfenbrenner, el desarrollo ocurre a través de procesos cada vez más complejos de interacción entre una 
persona en desarrollo y el ambiente inmediato cotidiano, procesos que son afectados por contextos más remotos de los cuales el individuo puede no estar consciente".

La consideración del desarrollo desde una perspectiva ecológica trae consigo la necesidad de comprender los procesos que interactúan y subyacen en las relaciones con otras personas y con los diversos contextos en que se desenvuelve.

Bronfenbrenner (1979), citado en Torrico, Santín, Andrés, Menéndez y López (2002, p. 46), indican que esto lleva a "(...) considerar el desarrollo humano como una progresiva acomodación entre un ser humano activo y sus entornos inmediatos (también cambiantes). Pero este proceso, además, se ve influenciado por las relaciones que se establecen entre estos entornos y por contextos de mayor alcance en los que están incluidos esos entornos". Esto es, se relacionan con diversos contextos o ambientes asociados al entorno físico, biológico, social, económico, político, estructural y estético (López y Escudero, 2003).

Dentro de estos contextos se encuentran el hogar, el lugar de trabajo, el vecindario, el sistema de educación y aspectos culturales e históricos que afectan la familia, la escuela y todo aquello con que interactúa la persona (Papalia et al., 2001).

Es así como la teoría ecológica propuesta por Bronfenbrenner involucra un modelo que propone una serie de sistemas contextuales relacionados, del más íntimo al más amplio, donde cada uno está contenido en el siguiente. De acuerdo con López y Escudero (2003), Papalia et al. (2001) y Torrico et al. (2002) estos son:

1. Microsistema: ambiente en el cual la persona interactúa cotidianamente con otras, en una relación cara a cara, por ejemplo, la familia, la escuela, la iglesia.

2. Mesosistema: implica la interacción de diferentes microsistemas que contienen a la persona en desarrollo, por ejemplo, el hogar, el lugar de trabajo, la iglesia, el vecindario, etc.

3. Exosistema: representa aquellos contextos o ambientes en los cuales la persona no participa directamente, pero que le afectan, como el comercio y la industria, jerarquía religiosa, sistema educativo, el gobierno, medios masivos de comunicación.

4. Macrosistema: involucra el micro, meso y exosistema. Este se relaciona con la ideología, valores, normas y patrones institucionales de cada cultura.

5. Cronosistema: afín con los efectos del tiempo en otros sistemas de desarrollo como la familia, el empleo, guerras, migración, entre otros.

La comprensión de los diversos sistemas en que una persona se desarrolla representa un insumo importante para la comprensión de su salud. Así, con la finalidad de favorecer su 
URL: http://www.una.ac.cr/educare

CORREO: educare@una.cr

bienestar y su capacidad para autogestionar su salud, podrían reconocerse elementos en los cuales se requiera intervenir de forma directa o indirecta, vinculados con aspectos de índole contextual o ambiental sobre los cuales pueda ejercer cierto control para hacer frente, de manera adecuada, a las demandas que la vida le plantea.

\section{La promoción de la salud: un modelo para sustentar la acción orientadora}

Una intervención orientadora para la salud requiere de un modelo que posibilite la operacionalización de los presupuestos del modelo biopsicosocial y la teoría ecológica, basados en la relación dialéctica entre la persona y su entorno, pues no se debe dejar de lado la realidad social en que se sitúa la persona, ya que su marco contextual estimula o dificulta su desarrollo y aprendizaje (Adame, Álvarez y Bisquerra, 2001).

Promoción de la salud es un concepto de reciente aplicación en el ámbito de salud, congruente con el nuevo posicionamiento epistemológico respecto a la salud y la enfermedad, el biopsicosocial. Este, para su abordaje, requiere de nuevas y diversas estrategias más allá de un tratamiento farmacológico para curar una enfermedad.

A mediados de la década de los 80, la Organización Mundial de la Salud (OMS) definió la promoción de la salud como "(...) el proceso mediante el cual la gente aumente el control sobre su salud y a la vez la mejore. Para alcanzar un estado de completo bienestar físico, mental y social, un individuo o un grupo debe ser capaz de identificar y llevar a cabo sus aspiraciones, satisfacer sus necesidades y cambiar o enfrentar el entorno (...)" (OMS, 1986, p. 1 citada en Marks et al., 2008, pp. 4-5).

El modelo de promoción de la salud promueve una serie de acciones que favorecen, en las personas y las sociedades, mayor control de los ámbitos personal, ambiental, contextual y de las condiciones de vida, lo que contribuye al estado de bienestar personal y social.

Se les otorga un rol protagónico a las personas y a las comunidades, debido a que implica su fortalecimiento en aras de que sean responsables de reconocer sus necesidades y autogestionar su salud, mediante acciones para enfrentarlas y satisfacerlas.

Refiere, entonces, a un posicionamiento centrado en el fortalecimiento de las capacidades individuales y colectivas; pues este modelo toma como punto de partida favorecer la salud integral:

(...) el compromiso de enfrentarse a los desafíos de reducir las desigualdades, incrementar el ámbito de la prevención y ayudar a los ciudadanos a afrontar sus circunstancias, supone favorecer la participación de la población, el fortalecimiento de los servicios de salud comunitarios (...) además la creación de entornos saludables, en los que las personas estén mejor preparadas para cuidar de sí mismas y ofrecerse apoyo mutuo en la resolución y superación de los problemas de salud (...) (Organización Panamericana de la Salud [OPS], 1996, p. 29). 
La promoción de la salud articula la responsabilidad personal con la de instituciones u organizaciones sociales, mediante políticas, programas y proyectos que propicien mejores condiciones personales, sociales y ambientales en procura de fortalecer la salud. Esta responsabilidad se traduce en acciones formadoras de:

(...) un proceso político y social que busca incidir en las condiciones sociales, ambientales y económicas, a fin de disminuir el efecto que estos factores pueden tener en el ámbito público e individual (...) por ello, sólo en la medida en que se concibe la salud como construcción colectiva, podrán controlarse las consecuencias de la globalización, de la inequidad, de la explotación en el trabajo, de las desigualdades de clase y género, entre otros aspectos (...). (Carmona, Rozo y Mogollón, 2005, pp. 71-72)

Como acción política y social, este modelo representa una maniobra unificada con la que se afecta, de manera simultánea, el ámbito personal, social, ambiental y económico. Concerniente a tres mecanismos integrados en los que la OPS (1996) ha denominado marco de la promoción de la salud:

- Autocuidado o decisiones y acciones que el individuo toma en beneficio de su propia salud. Supone estimular las elecciones saludables, ante las cuales, las creencias, la información adecuada y encontrarse en un entorno manejable para la persona desempeñan un papel importante en su estado de salud.

- Ayuda mutua o acciones que las personas realizan para ayudarse unas a otras. Se prestan apoyo emocional mutuamente, y comparten ideas, información y experiencias. Ello puede surgir en el contexto de la familia, el vecindario, la organización voluntaria o grupos de autoayuda. De manera que la persona puede vivir relacionada y, al mismo tiempo, conservar su independencia en materia de salud.

- Entornos sanos o creación de las condiciones y entornos que favorecen la salud. Se refiere a alterar o adaptar los entornos sociales, económicos o físicos de forma que ayuden a conservar y aumentar la salud. Implica que existan políticas y prácticas que propicien a las personas un medio ambiente saludable.

Estos tres mecanismos establecen que la promoción de la salud actúa en lo personal, en el fortalecimiento de las capacidades, para la toma de decisiones en las personas, de manera que sus decisiones sean saludables. 
URL: http://www.una.ac.cr/educare

CORREO: educare@una.cr

En el plano social se construye, mediante políticas y prácticas sociales, un andamiaje que propicie condiciones de salud adecuadas para que favorezcan la salud integral, no solo desde una perspectiva médica; además que existan redes sociales de apoyo disponibles para todas las personas independientemente de la condición de salud que presenten.

La intervención orientadora bajo el modelo de promoción de la salud promueve una serie de acciones que favorecen, en las personas y las sociedades, mayor control de los diversos elementos que intervienen en su salud, lo que contribuye a visualizarse en un estado de bienestar personal y social y enfatiza en respuestas vinculadas a la prevención y promoción, más que en una acción remedial ante la crisis, la enfermedad o las problemáticas psicosociales.

\section{La promoción de la salud mental como énfasis en la acción orientadora}

La posición epistemológica asumida respecto a la salud pone de manifiesto que este campo de actuación no puede seguir siendo exclusivo para profesionales de las ciencias de la salud, sino que implica la incorporación de nuevos actores sociales. Dicha participación estará sujeta a la naturaleza de los objetivos, principios y funciones de cada disciplina, así como de los novedosos aportes que surjan producto del trabajo inter o transdisciplinario (Gavilán, 2006).

La promoción de la salud, al abarcar aspectos individuales y del entorno, demanda “(...) la participación de una gama mucho más amplia de intervenciones y actores que la que conlleva el modelo tradicional de la medicina que se centra en capacitar a los especialistas para que devuelvan la función a los individuos" (OMS, 2004, p. 18).

La orientación cuenta con los referentes teóricos y metodológicos necesarios para comprender y favorecer el desarrollo socioemocional de las personas en las diversas etapas del desarrollo, en los diferentes contextos y situaciones en que estas se encuentren, pues "(...) enfatiza lo afectivo y se centra en el individuo como un todo que interactúa con su medio socio-cultural e histórico. Esta orientación, como desarrollo de fortalezas humanas, guiadas evolutivamente, permite trabajar con cualquier problemática y cualquier población, ya que lo que busca es rescatar las habilidades y destrezas que posee la persona, a pesar de su problema" (Bonilla, 1993, p. 57).

Así el aporte de la orientación en el campo de la salud queda circunscrito a la salud mental, concepto que alude al estado de bienestar sustentado en la toma de conciencia de las propias aptitudes y la capacidad de afrontamiento de las presiones normales de la vida (OMS, 2004).

Su aporte como disciplina resulta valioso pues "(...) la salud mental es esencial para el bienestar y funcionamiento de los individuos (...) como una parte indivisible de la salud general, contribuye a las funciones de la sociedad y tiene un efecto sobre la productividad general" (OMS, 2004, p. 25). 
Tanto en contextos y con poblaciones tradicionales (centros educativos, universidades, sistema penitenciario) como no tradicionales (empresas privadas, organizaciones no gubernamentales, organizaciones comunitarias, entre otros) la intervención orientadora para la promoción de la salud mental cobra relevancia para coadyuvar al bienestar personal y social, hecho que demanda la delimitación del quehacer de la persona orientadora en el nuevo rol que le implica este campo de intervención.

\section{La persona orientadora como promotora de salud mental}

Los presupuestos teóricos asumidos para comprender la salud mental y la intervención en este campo de actuación ubican a la persona orientadora ante nuevas demandas de sentido en su ejercicio profesional como promotora de salud mental.

Su rol enfatiza en hacer conscientes a las personas de los obstáculos personales y contextuales que impiden su plena realización personal, de manera que esto le lleve a la acción para hacer frente, de forma adecuada, a los desafíos de la vida (Adame et al. 2001).

Así, las personas disponen de diferentes opciones para ejercer un mayor control sobre su salud mental y sobre las situaciones con que interactúa en su medio social, lo cual le posibilita insumos para optar por todo aquello que le resulte saludable (Tobón, 2003).

En el marco de promoción de la salud propuesto por la OPS (1996), el aporte está vinculado a los mecanismos referidos al autocuidado y a la mutua ayuda, esta última mediante la articulación de las redes sociales con que se cuente y con el trabajo de grupos de apoyo, de esta manera logra generar oportunidades para que las personas mantengan o mejoren, en este caso, su salud mental.

Al enfocarse en el autocuidado, fortalece habilidades y destrezas personales que posibiliten la toma de decisiones saludables y el empoderamiento para la autogestión de la salud mental. Al facilitar procesos de ayuda mutua, promueve que las personas en un grupo encuentren apoyo, contención y puedan vislumbrar alternativas ante situaciones vitales que conlleven a su bienestar físico, emocional y social.

En síntesis la acción orientadora promotora de salud mental debe favorecer la toma de conciencia en las personas mediante el análisis de los factores personales, sociales, económicos, políticos, religiosos, entre otros, que caracterizan su estilo de vida e intervienen en su salud integral. De acuerdo con Tobón (2003), tanto en el abordaje individual como grupal, se deben considerar de igual forma los aspectos internos y los externos a la persona, entre estos:

- Conocimientos: referidos al acceso a la información que facilite la toma de decisiones y el asumir prácticas saludables.

- Voluntad: relacionada con los valores, creencias y motivaciones personales que movilizan a la persona frente a las prácticas y decisiones saludables versus las conductas de riesgo que pueda asumir. 
URL: http://www.una.ac.cr/educare

CORREO: educare@una.cr

- Actitudes: vinculadas a principios, valores y creencias que sustentan una posición personal frente alguna situación que resulte demandante.

- Hábitos: relacionados con rutinas incorporadas al estilo de vida que pueden beneficiar o perjudicar la salud.

- Cultura: vinculada a las tradiciones, creencias y representaciones sociales respecto a la salud y las enfermedades propias de un contexto en particular, que pueden limitar la adquisición de nuevos conocimientos y prácticas que resulten saludables.

- Género: asociado al impacto que tienen los roles estereotipados de género del sistema patriarcal, en la toma de decisiones y prácticas saludables en que incurren tanto hombres como mujeres, con lo que puedan verse limitados en la autogestión de su salud.

- Ciencia y tecnología: refiere a los avances científicos y tecnológicos que aumentan las posibilidades para el cuidado de la salud.

- Físico, económico, político y social: alude a aspectos como infraestructura sanitaria, políticas de la salud pública, inversión económica en salud y la generación de contextos saludables.

Los aspectos antes señalados representan elementos determinantes del proceso de salud-enfermedad. Su consideración en la intervención orientadora facilita, en las personas, una visión integral de sus circunstancias vitales y fomenta la toma de decisiones adecuada, basada en un análisis multifactorial que brinda insumos para la autogestión de su bienestar personal y social.

En su función como promotora de salud mental, la persona orientadora requiere poner en práctica algunos elementos que le permitan ampliar su práctica para atender satisfactoriamente las demandas de este campo de actuación y, a la vez, aportar nuevos conocimientos a la disciplina. De acuerdo con Gavilán (2006) y Gordillo (1988), resulta indispensable:

- Un modelo comprensivo con mayor influjo social: enfatiza en los aspectos sociales que le permitan hacer lectura del contexto y la comprensión de los elementos de tipo político, social, cultural y económico que concurren en los diversos sistemas sociales en que interactúan las personas. Esto le brinda una visión sociológica y antropológica para la comprensión del estilo de vida de las poblaciones que atiende.

- Modelos teóricos comprensivos: implica la construcción de una posición ecléctica basada en la integración de diversas corrientes teóricas que sustenten la intervención. Supone articular los elementos comunes de las teorías ya existentes para diseñar prototipos de 
acción que permitan atender situaciones particulares de manera exitosa, mediante la intervención, de manera que se establece una relación dialéctica entre teoría y práctica.

- Modelo integral de prevención: Permite anticiparse a la ocurrencia de problemas o reducir su riesgo mediante estrategias de intervención. Requiere de la investigación aplicada para conocer la realidad concreta en que ejerce su labor profesional y, a la vez, para diseñar, ejecutar y evaluar las metodologías más propicias para el abordaje de una población y situación particular.

- Inclusión de saberes disciplinarios, interdisciplinarios y transdisciplinarios: permite la integración de los referentes conceptuales de diversas disciplinas y áreas del saber, que confluyan para lograr en la práctica mejores intervenciones ante situaciones particulares. Implica la inclusión de los referentes teóricos y metodológicos de diversas disciplinas científicas en el trabajo inter y transdisciplinario. A la vez, la inclusión de estrategias y técnicas propias de la sabiduría de los pueblos.

La persona orientadora, al articular dichos elementos para ampliar su práctica profesional en el campo de la salud mental, puede establecer, de manera adecuada, la relación entre teoría y práctica, hecho que le da posibilidades para ejercer su rol profesional con cientificidad de forma efectiva en este campo de intervención.

En la medida en que -por formación inicial o por formación permanente- las personas que ejerzan la orientación cuenten con los insumos teóricos, metodológicos e instrumentales no solo para comprender los procesos de desarrollo socioemocional de las personas, sino también para operacionalizar un posicionamiento epistemológico centrado en la relación dialéctica persona-entorno, la intervención podrá ser acorde a las demandas de un contexto social complejo en el campo de actuación que constituye la salud mental.

Incursionar en el campo de la salud mental le demanda, a las instituciones con escuelas formadoras de profesionales en orientación, un análisis conducente a la inclusión de nuevos contenidos en la estructura curricular del plan de estudio de la carrera, vinculados a un posicionamiento epistemológico para la comprensión de la salud que enfatice no solo en aspectos personales, sino también contextuales y ambientales. Por otra parte, a las personas que ya ejercen la profesión, interesadas en este, un proceso de autoactualización para ampliar su práctica profesional, enfocado en una perspectiva contextual de la salud mental.

\section{Consideraciones finales}

La complejidad de las circunstancias sociales en que se desarrollan las personas tiene implicaciones en su bienestar personal y colectivo. Los factores de tipo económico, político, 
URL: http://www.una.ac.cr/educare

CORREO: educare@una.cr

social, familiar, étnico, religioso, entre otros, representan riesgos importantes para la salud de las personas, hecho que trae consigo trascender la comprensión de la salud como sinónimo de la ausencia de enfermedad.

Esta consideración representa un cambio paradigmático para el abordaje de la salud, pues se concibe la salud como proceso multifactorial y como estado de bienestar con atributos físicos, sociales, axiológicos, culturales, que están interrelacionados, y que a su vez requiere la incursión de nuevos actores sociales, entre estos las personas profesionales de la orientación, en este campo de intervención.

La orientación como disciplina, al contar con referentes teóricos, metodológicos e instrumentales para la comprensión del desarrollo socioemocional de las personas, tiene un aporte significativo que realizar, al centrar su intervención en el fortalecimiento de capacidades individuales y colectivas que faciliten el mantenimiento o mejoramiento de la salud.

Con un posicionamiento epistemológico basado en la relación dialéctica individuoentorno y de la salud como proceso biopsicosocial, la persona orientadora se asume como promotora de salud, en particular de salud mental, al tener como propósito el empoderamiento personal y colectivo mediante la toma de conciencia de la complejidad de las circunstancias vitales, de las fortalezas y habilidades que permitan a las personas vislumbrar alternativas para enfrentarlas.

Incursionar en la salud mental como campo de intervención representa una serie de demandas de sentido para el ejercicio profesional vinculadas a la ampliación de la práctica profesional. Mediante un modelo comprensivo con mayor influjo social, modelos teóricos comprensivos, un modelo de prevención y la inclusión de saberes, la persona orientadora puede construir prototipos de acción eficientes para abordar las necesidades particulares de las poblaciones que atiende.

A la vez, articular la valoración de factores tanto internos como externos que intervienen en el proceso de salud mental, para que las personas logren comprender integralmente su realidad, tomen conciencia de sus recursos y posibilidades y cuenten con insumos para optar por aquello que les resulte saludable.

Los argumentos sobre los que se ha reflexionado constituyen solo una propuesta producto del interés personal y profesional. Como tal, se espera que sirva de insumo para la discusión y delimitación de lo que se considera un campo de intervención emergente para la orientación. 


\section{Referencias}

Adame, M.T., Álvarez, M. y Bisquerra, R. (2001). Principios, objetivos y funciones de la orientación. En R. Bisquerra (Coord.). Modelos de orientación e intervención psicopedagógica (pp. 41-54). España: Ciss Praxis.

Bonilla, F. S. (marzo, 1993). Orientación de poblaciones abusadas. Revista de Ciencias Sociales Universidad de Costa Rica, 59, 53-62.

Carmona, L. D., Rozo, C. M. y Mogollón, A. S. (enero-junio, 2005). La salud y la promoción de la salud: Una aproximación a su desarrollo histórico y social. Revista Ciencias de la Salud, 3(1), 62-77.

Flórez, L. (2007). Psicología social de la salud. Promoción y prevención. Colombia: Editorial El Manual Moderno.

Gavilán, M. G. (2006). La formación de orientadores en contextos complejos con especial referencia a Latinoamérica. En Z. R. Vargas (s. f.). Antología del curso Orientación para la vida adulta. Maestría Profesional en Orientación, San José, Costa Rica: Universidad de Costa Rica.

González, M. y García, M. (1994). Psicología de la salud y educación para la salud. El contexto escolar como marco en prevención y educación para la salud. Revista Aula, $6,127-142$.

Gordillo, M. V. (1988). Manual de orientación educativa. España: Editorial Grupo Anaya Comercial.

López, S. y Escudero, V. (2003). Familia, evaluación e intervención. España: Editorial CCS.

Marks, D. F., Murray, M. y Evans, B. (2008). Psicología de la salud: Teoría, investigación y práctica. México: Manual Moderno.

Morago, P. (2006). Tratamiento comunitario asertivo: Prácticas, consideraciones éticas y rol del trabajador social. Cuadernos de Trabajo Social, 19, 7-23.

Oblitas, L. A. (2006). Psicología de la salud y calidad de vida. México: Thomson Learning

Organización Mundial de la Salud (OMS). (2004). Promoción de la salud mental: conceptos, evidencia emergente, práctica. Recuperado de http://www.who.int/mental_health/ evidence/promocion_de_la_salud_mental.pdf

Organización Panamericana de la Salud (OPS). (1996). Promoción de la salud: Una antología. Estados Unidos.

Papalia, D. E., Wendkos, S. y Duskin, R. (2001). Desarrollo humano. Bogotá: McGraw-Hill 
URL: http://www.una.ac.cr/educare

CORREO: educare@una.cr

Tobón O. (enero-diciembre, 2003). El autocuidado una habilidad para vivir. Revista Hacia la Promoción de la Salud, 8, 1-12. Recuperado de http://promocionsalud.ucaldas.edu.co/ downloads/Revista 8_5.pdf

Torrico, E., Santín, C., Andrés, M., Menéndez, S. y López, M. J. (junio, 2002). El modelo ecológico de Bronfenbrenner como marco teórico de la psicooncología. Anales de Psicología, 18(1), 45-59.s Recuperado de http://redalyc.uaemex.mx/src/inicio/ArtPdfRed.jsp?iCve=167181 03jsp?iCve $=16718103$

\section{Cómo citar este artículo en APA:}

Garita, A. V. (2013). La orientación para la salud mental: Reflexiones para delimitar un campo de intervención profesional. Revista Electrónica Educare, 17(2), 55-68. Recuperado de http://www.revistas.una.ac.cr/index.php/EDUCARE/issue/current

Nota: Para citar este artículo en otros sistemas puede consultar el hipervínculo "Como citar el artículo" en la barra derecha de nuestro sitio web:

http://www.revistas.una.ac.cr/index.php/EDUCARE/index 